Discrete Optimization

\title{
Solving robust bin-packing problems with a branch-and-price approach
}

\author{
Xavier Schepler ${ }^{\mathrm{a}, \mathrm{b}, *}$, André Rossi ${ }^{\mathrm{a}, \mathrm{c}}$, Evgeny Gurevsky ${ }^{\mathrm{d}}$, Alexandre Dolgui ${ }^{\mathrm{e}}$ \\ a LERIA, Université d'Angers, France \\ ${ }^{\mathrm{b}}$ Recommerce RED, France \\ ${ }^{c}$ LAMSADE, Université Paris-Dauphine, PSL, France \\ d LS2N, Université de Nantes, France \\ e IMT Atlantique, LS2N, Nantes, France
}

\section{A R T I C L E I N F O}

\section{Article history:}

Received 5 August 2020

Accepted 25 May 2021

Available online 1 June 2021

\section{Keywords:}

Packing

Robustness

Sensitivity analysis

Uncertainty

Stability radius

Relative resiliency

Dantzig-Wolfe decomposition

Column generation

Branch-and-price

\begin{abstract}
A B S T R A C T
One-dimensional bin-packing is a well-known combinatorial optimization problem which is strongly NPhard. It consists of allocating a given set of items of different sizes into bins of the same capacity to minimize the number of bins used. The capacity of each bin cannot be exceeded. This paper deals with some variants of this problem to take into account the cases when there are items with uncertain sizes. The goal is to obtain robust solutions taking into account possible variations of item sizes around their nominal values. First, two robust approaches are considered which are based on a stability radius calculation, to ensure that the stability radius, measured either with the Manhattan or Chebyshev norm, is not below a given threshold. Then, a complementary robust approach is applied which is based on a relative resiliency calculation. To solve to optimality these robust variants of the bin-packing problem, a compact 0-1 linear programming formulation, which is also valid for the standard bin-packing problem, is introduced. Then, a Dantzig-Wolfe decomposition is suggested in order to provide a set-cover reformulation with a stronger linear relaxation, but an exponential number of columns. Finally, to obtain integer optimal solutions, a branch-and-price algorithm is developed, whose linear relaxation of the set-cover formulation is solved by a dynamic column generation. Numerical experiments are conducted on adapted benchmark sets from the literature. The performance of the branch-and-price algorithm allows us to investigate what protection against uncertainty is offered by each approach, and at which cost of robustness.
\end{abstract}

(c) 2021 Elsevier B.V. All rights reserved.

\section{Introduction}

For the standard bin-packing $(B P)$ problem, one-dimensional items of different sizes have to be assigned to a minimum number of identical one-dimensional bins. The size of each item and the capacity of a bin are assumed to be known in advance. Nevertheless, in real-life applications of $B P$, the sizes of items often have random deviations from the nominal values. For example, in hospital administration (see Cardoen, Demeulemeester, \& Beliën, 2010; Lamiri, Xie, Dolgui, \& Grimaud, 2008), one of the most important optimization issues which can be modeled as BP problems, is the daily planning of surgeries. The surgery durations are likely to change. A smaller number of operating rooms used per day reduces the cost of dealing with their preparation. The context of

\footnotetext{
* Corresponding author at: LERIA, Université d'Angers, France.

E-mail address: xavier.schepler@gmail.com (X. Schepler).
}

$B P$ under uncertainty appears also in the domain of supply-chain management. For example, for the problem of logistics capacity planning (see Crainic, Gobbato, Perboli, \& Rei, 2016), where it is necessary to predict four to six months in advance the allocation of goods to a minimum number of containers of different capacities. The volumes of delivered goods have random deviations around the planned values. BP models are also used in the design of paced assembly lines. The problem is known in the literature as an assembly balancing problem of type 1 (see Battaïa \& Dolgui, 2013; Boysen, Fliedner, \& Scholl, 2007), where a given set of production tasks, some involving issues of precedence, has to be assigned to a minimum number of identical workstations. The so-called cycle time constraint expresses a limit on a station capacity. In the case of non-fully automated lines, there is a subset of manual tasks, whose processing times may vary depending on operator skills and random performances. The assembly line balancing problem of type 1 is a $B P$ problem with precedence constraints. Another case of $B P$ problems under uncertainty is the one dimensional cutting 
stock problem, as in Alem, Munari, Arenales, and Ferreira (2010), where the demand or item sizes can be uncertain. A robust machine availability problem in a parallel machine environment with uncertain task durations in Song, Kowalczyk, and Leus (2018) is another example. The latter two articles are discussed in more detail in Section 3, dedicated to the literature review.

Probabilistic models are often used to consider uncertainty. Nevertheless, this approach is not always possible because of the absence of reliable historical data in order to establish adequate probability distributions as, for example, in the case of the initial design of the aforementioned assembly lines. Only a subset of tasks with uncertain processing times can be identified by decision makers at the line design stage. In order to overcome this difficulty, Rossi, Gurevsky, Battaïa, and Dolgui (2016), motivated by the work of Sotskov, Dolgui, and Portmann (2006), proposed a new approach, based on the stability radius optimization. For any given solution, its stability radius is obtained as the maximal magnitude of variations from their nominal values of the uncertain task times for which the solution feasibility (or optimality) is preserved. In other words, studying the simple assembly line balancing problem with a fixed number of workstations, the authors search for solutions which maximize the stability radius. The well-known norms $\ell_{1}$ and $\ell_{\infty}$ are used to calculate the stability radius. First, the authors show that if the $\ell_{1}$ norm is used, then the minimum idle time $^{1}$ among all the workstations having at least one uncertain task, has to be maximized. Second, they also show that if the $\ell_{\infty}$ norm is employed, a duration equal to the stability radius, added to the duration of each uncertain task, has to be maximized. Thus, from a managerial point of view, it is necessary to search for a feasible task assignment having the maximal value of the mentioned above indicators, since this leads to the greatest protection against uncertainty.

In this paper, we develop a branch-and-price method applied to the problems, which are inverse to those addressed in Rossi et al. (2016). By considering a BP problem, therefore excluding precedence constraints, the problem consists of allocating a given set of items to a minimum number of bins so that the stability radius of the solution is not less than a specified threshold, which determines a desired level of robustness. Then, to overcome a possible limitation of the previous approach, in which the variation of an uncertain size is not related to its nominal value, another robust approach based on the concept of relative resiliency proposed by Pirogov (2019), is developed. The relative resiliency of a solution is the proportion by which each uncertain size can be increased without losing solution feasibility.

Hereafter, the robust $B P$ problem ensuring that the stability radius calculated for the $\ell_{1}$ norm (resp. the $\ell_{\infty}$ norm) is not less than $r$ is denoted as $R B P_{1}(r)$ (resp. $R B P_{\infty}(r)$ ), and the robust $B P$ problem ensuring the relative resiliency is not less than $\alpha$ is denoted as $R B P_{r r}(\alpha)$. These three problems can be simply stated as follows.

We want to pack a given set of one-dimensional items whose nominal sizes are known into a minimum number of onedimensional identical bins of the same given capacity. A known subset of these items have uncertain sizes, which can vary after the allocation of items to bins. The solution must remain feasible for real item sizes, provided that the variations of uncertain sizes are bounded as follows:

- with $R B P_{\infty}(r)$, each uncertain size can be increased by a maximum of $r$,

- with $R_{B P}(\alpha)$, each uncertain size $w_{i}$ can be increased by a maximum of $\alpha w_{i}$,

\footnotetext{
${ }^{1}$ Idle time is the difference between the cycle time and the effective load of the considered workstation.
}

- with $R B P_{1}(r)$, in any bin with at least one uncertain-size item, the sum of all uncertain sizes' variations is at most $r$.

Given these conditions, a solution to any instance of $R B P_{\infty}(r)$ (resp. $\left.R B P_{r r}(\alpha)\right)$ can be obtained by solving an instance of $B P$, in which each uncertain size $w_{i}$ is increased by $r$ (resp. $\alpha w_{i}$ ), to reserve free space. Therefore, $R B P_{\infty}(r)$ and $R B P_{r r}(\alpha)$ can both be straightforwardly reduced to $B P$. However, this is not the case with $R B P_{1}(r)$, as it requires a quantity of free space not lesser than $r$ in each bin containing at least one item of uncertain size.

Note that, a solution to $B P$ has a stability radius (resp. relative resiliency) at least equal to $r$ (resp. $\alpha$ ) if and only if a quantity $r$ (resp. $\alpha w_{i}$ ) of free space is reserved for each item $i$ of uncertain size $w_{i}$ in its assigned bin. Similarly, a solution to BP has a stability radius at least equal to $r$ if and only if a quantity $r$ of free space is reserved in each bin containing at least one uncertain item. Formal definitions of stability radius, relative resiliency, and proofs of the properties stated above are provided in Appendix A.

The remainder of this paper is organized as follows. Section 2 provides a literature review about column-generation based approaches for variants of $B P$ under uncertainty and branchand-price algorithms for $B P$. Section 3 presents the details of the adapted branch-and-price method for the proposed set-cover formulation valid for $R B P_{1}(r), R B P_{\infty}(r), R B P_{r r}(\alpha)$ and $B P$. Section 4 is devoted to numerical experiments, in which we evaluate the performance of the proposed branch-and-price algorithm and we investigate what protection against uncertainty is offered by each approach and at which cost of robustness. Section 5 concludes the paper and gives some perspectives for future work.

\section{Literature review}

We first consider two papers introducing column-generation based algorithms to tackle variants of $B P$ under uncertainty, then the literature about branch-and-price algorithms designed to solve the set-cover formulation of BP provided in Section 3.2.

Song et al. (2018) address the robust machine availability problem in a parallel machine environment, which in its deterministic version coincides with $B P$. Using the methodology proposed by Bertsimas and Sim (2004), they introduce a robust version with budgeted uncertainty, where at most $\Gamma$ jobs can deviate from their nominal processing times, which are assumed to belong to symmetric and bounded intervals. They propose a set-cover formulation with an exponential number of columns. The formulation is solved by a branch-and-price algorithm, with the Ryan and Foster branching scheme, and zero-suppressed binary decision diagrams to handle a pricing problem more complicated than the one occuring with $B P$. They perform numerical experiments on a new set of randomly generated instances. Results show that their algorithm can handle instances with up to 180 items within a time limit of 1200 seconds, provided that either the value of $\Gamma$ is small, or the size of each item is in the range $\{1, \ldots, 20\}$.

Alem et al. (2010) address a stochastic variant of the one dimensional cutting stock problem, where demand is a random variable. The authors propose a two stage stochastic non-linear program, where the first stage decisions are the numbers of objects to be cut according to a cutting pattern, and the second stage decisions are the numbers of holding or backordering items. The objective is to minimize the expected costs due to waste and holding or backordering penalties. A column-generation based algorithm is introduced to solve the linear relaxation of the formulation, and heuristics allow integer solutions to be obtained. Numerical experiments are performed to investigate the value of the stochastic solution and the expected value with perfect information. The results show that the two-stage stochastic approach represents a better modeling framework than the alternative wait-and-see and 
expected value frameworks, even under small variations in the parameters of the problem.

There is abundant literature about branch-and-price algorithms, including general studies, for example by Barnhart, Johnson, Nemhauser, Savelsbergh, and Vance (1998), Vanderbeck (2000), and Desrosiers and Lübbecke (2011), as well as many other application-related studies, in various domains, such as cutting and packing (Delorme, Iori, \& Martello, 2016), vehicle routing (Fukasawa et al., 2006), production planning (Alfandari, Plateau, \& Schepler, 2015), etc. The theories and concepts behind branch-andprice algorithms are covered in the Ph.D. thesis by Gamrath (2010).

We focus on two branch-and-price algorithms similar to the one proposed in Section 3 designed to solve the set-cover formulation of $B P$, which corresponds to the set-cover formulation proposed in this paper, when there is either no uncertain item or all items are uncertain, or $r$ is equal to zero. These are the ones by Vance, Barnhart, Johnson, and Nemhauser (1994) and by Gamrath et al. (2016).

The first branch-and-price algorithm for the set-cover formulation of BP was proposed by Vance et al. (1994). The first-fit decreasing heuristic initializes the solving process with a primal solution. The branch-and-price algorithm relies on the Ryan and Foster branching scheme, which selects one of the fractionally packed pairs of items for branching, and performs branching so that either the items are packed in the same bin, or in two different bins. In the first case, when items have to be packed in the same bin, the structure of the subproblem is preserved, as it suffices to merge the items. In the second case, the structure of the subproblem is altered, with the presence of a conflict constraint, forbidding the items in conflict to be packed together. When considering pairs of items for branching, the one with the largest total size is selected. Nodes of the search tree without any conflict constraint are explored first, as their subproblem can be solved by a more efficient algorithm. Vance et al. (1994) proposed solving the subproblem without conflict constraint, that is, the pseudo-polynomial 0-1 knapsack problem, with the Horowitz \& Sahni (1974) algorithm, having a worst case temporal complexity in $\mathcal{O}\left(\min \left\{2^{n / 2}, n\right.\right.$. $W\}$ ), potentially better than the one of the classical dynamic programming algorithms, in $\mathcal{O}(n \cdot W)$, described by Kellerer, Pferschy, and Pisinger (2004). When each item is in conflict with at most one other item, Vance et al. (1994) use an adapted version of the algorithm by Horowitz and Sahni (1974). Otherwise, in the presence of conflict constraints, the subproblem becomes strongly NP-hard, and it is addressed with an integer linear program solver.

The branch-and-price algorithm proposed by Gamrath et al. (2016) also uses a branching scheme based on the Ryan and Foster branching scheme. Nevertheless, it differs from the one by Vance et al. (1994) on several points. First, one of the most unfeasible pairs of items is selected for branching, that is, the one which provides a sum value closest to 0.5 . Second, the node which is selected for exploration is the one which provides the best estimation value of the progress toward integer feasibility, relative to its degradation of the objective function. Third, since this branch-andprice algorithm is provided as an example with the SCIP Optimization Suite, it relies on its integer linear program solver to address the subproblem. Last, for the same reason, it uses primal heuristics of the SCIP Optimization Suite, described in Berthold (2008) and Achterberg, Berthold, and Hendel (2012). This includes more than forty very fast heuristics, generally requiring only a few milliseconds, even with the largest $B P$ instances. They are called repetitively during the exploration of the search tree. The branch-andprice algorithm that we propose is presented in the next section, and some of the concepts involved, such as dynamic column generation, are covered in more detail.

\section{A branch-and-price algorithm}

We are given a set $K$ of one-dimensional identical bins of capacity $W$, a set $V=\{1,2, \ldots, n\}$ of one-dimensional items, a nominal size $w_{i}$ for each item $i \in V$, and a set $\widetilde{V} \subseteq V$ of items of uncertain size. An allocation of the set of items to the set of bins is called a feasible solution to $B P$ if each item is assigned to exactly one bin such that the capacity constraints are satisfied. We use the following decision variables: $x_{i k}$ is set to one if item $i$ is assigned to bin $k$, and zero otherwise, and $y_{k}$ is set to one if bin $k$ is used and zero otherwise.

We introduce a branch-and-price algorithm for $R B P_{1}(r)$ derived from the ones proposed by Vance et al. (1994) and by Gamrath et al. (2016) for $B P$. This algorithm can also be used for $B P$, which corresponds to $\operatorname{RBP}_{1}(r)$ when there is no uncertain items, and, therefore, for $R B P_{\infty}(r)$ and $R B P_{r r}(\alpha)$, as explained below.

$R B P_{\infty}(r)$ can be formulated as follows.

$\min \sum_{k \in K} y_{k}$

s.t. $\quad \sum_{k \in K} x_{i k} \geq 1, \quad \forall i \in V$,

$\sum_{i \in \widetilde{V}}\left(w_{i}+r\right) x_{i k}+\sum_{i \in V \backslash \widetilde{V}} w_{i} x_{i k} \leq W y_{k}, \quad \forall k \in K$,

$y_{k} \in\{0,1\}, \quad \forall k \in K$,

$x_{i k} \in\{0,1\}, \quad \forall i \in V, \quad \forall k \in K$.

Where (1a) minimizes the number of open bins, (1b) assigns each item to at least one bin, (1c) enforces the bin capacity. Here, the size of all the uncertain items has been increased by the positive constant $r$, as per Property 4 presented in Appendix A, and (1d)-(1e) are domain constraints.

$R B P_{r r}(\alpha)$ can be formulated using the same BP model, but where constraints (1c) should be replaced with:

$\sum_{i \in \widetilde{V}} w_{i}(1+\alpha) x_{i k}+\sum_{i \in V \backslash \widetilde{V}} w_{i} x_{i k} \leq W y_{k}, \quad \forall k \in K$.

In this constraint, the size of all the uncertain items is multiplied by $1+\alpha$, where $\alpha$ is a positive constant, as per Property 5 presented in Appendix A.

\subsection{Compact formulation of $R B P_{1}(r)$}

The compact formulation of $R B P_{1}(r)$ uses an additional decision variable: $a_{k}$ is set to one if bin $k$ contains at least one uncertainsize item, and zero otherwise.

$R B P_{1}(r)$ can be formulated as follows.

$\min \sum_{k \in K} y_{k}$

s.t. $\quad \sum_{k \in K} x_{i k} \geq 1, \quad \forall i \in V$,

$x_{i k} \leq a_{k}, \quad \forall i \in \widetilde{V}, \quad \forall k \in K$,

$\sum_{i \in V} w_{i} x_{i k}+r a_{k} \leq W y_{k}, \quad \forall k \in K$,

$y_{k} \in\{0,1\}, \quad \forall k \in K$, 
$a_{k} \in\{0,1\}, \quad \forall k \in K$,

$x_{i k} \in\{0,1\}, \quad \forall i \in V, \quad \forall k \in K$.

Constraints (2b) are the same as (1b). Constraints (2c) give to each variable $a_{k}$ the value 1 if bin $k$ contains at least one uncertain-size item. Constraints (2d) represent the bin capacity, taking into account the minimum desired level of robustness $r$ for the bins having uncertain-size items. This constraint is also a natural consequence of Property 6, presented in Appendix A. More specifically, this property pertains to bins that accommodate uncertain items. In the present formulation of $R B P_{1}(r)$, these bins are open $\left(y_{k}=1\right)$ and such that $a_{k}=1$. Objective (2a) is to minimize the number of bins used.

Note that, $B P$ is a particular case of $R B P_{1}(r)$, where there is no uncertain item $(\widetilde{V}=\emptyset)$, or all items are uncertain $(V=\widetilde{V})$, or, alternatively $r=0$. When there is no uncertain item, variables $a_{k}$ can be set to 0 , and we obtain a formulation of $B P$. When all items are uncertain, or $r=0$, variables $a_{k}$ can be set to 1 , and we also obtain a formulation of $B P$.

Besides, the linear programming relaxation of this compact formulation is expected to be weak, as shown by Vance et al. (1994) for their formulation of BP, which we extended. To check if a state-of-the-art solver is able to solve this compact formulation, we performed numerical experiments on adapted instances of Falkenauer (1996). We added $20 \%$ of uncertain-size items and we set $r$ equal to $10 \%$ of the bin capacity. Only 30 of the 80 adapted instances were solved by IBM CPLEX 12.7 within a time limit of one hour per instance, which motivated us to develop a more efficient approach.

\subsection{Set-cover reformulation of $R B P_{1}(r)$}

Another possibility is to use a set-cover formulation, which is obtained by applying the Dantzig-Wolfe decomposition principle (Dantzig \& Wolfe, 1960), in order to obtain in this case a much tighter lower bound on the optimal value of bins.

Once the constraints (2b) are dualized in a Lagrangian way (Lemaréchal, 2007), subsystem (2c) and (2d) decomposes into a subproblem for each bin $k \in K$. Let $\mathcal{B}$ be the family of the subsets of items that can be fit into one bin with respect to uncertain sizes and to the threshold $r$ on stability radius $\rho_{1}$. Each subset $B \in \mathcal{B}$ is defined by an indicator vector $x^{B}\left(x_{i}^{B}=1\right.$ iff item $i \in V$ is in set $\left.B\right)$ and associated with a binary variable $\lambda_{B}$ taking value 1 if the corresponding subset of items is selected to fill one bin.

The reformulation is:

$$
\min \sum_{B \in \mathcal{B}} \lambda_{B}
$$

s.t. $\quad \sum_{B \in \mathcal{B}} x_{i}^{B} \lambda_{B} \geq 1, \quad \forall i \in V$,

$\sum_{B \in \mathcal{B}} \lambda_{B} \leq K$

$\lambda_{B} \in\{0,1\}, \quad \forall B \in \mathcal{B}$.

Here, constraints (3b) replace constraints (2b), constraints (3c) limit the number of used bins to at most $K$, and all other constraints of the compact formulation are built into the definition of feasible sets $B \in \mathcal{B}$ of items. This problem will be referred to as the master problem. When $\widetilde{V}=\emptyset$ or $\widetilde{V}=V$ or $r=0$, we obtain the setcover formulation of $B P$, for which it is conjectured that the difference between the linear relaxation value at root node and the optimal solution value is always less than or equal to two (see, for example, Scheithauer \& Terno, 1997).

The set-cover formulation, which has an exponential number of columns, is addressed by a branch-and-price approach. Its principles are described in Section 3.3. At each node of a branch-andbound tree, its linear relaxation is solved by dynamic column generation. The master problem is restricted to a subset of columns, and its linear relaxation is solved by the simplex method. Then, a pricing subproblem is solved to check for the existence of an improving column, with a negative reduced cost. It consists in solving the following robust variant of the knapsack problem, denoted as $P S$ :

$\max \sum_{i \in V} \pi_{i} z_{i}$

s.t. $z_{i} \leq a, \quad \forall i \in \widetilde{V}$

$\sum_{i \in V} w_{i} z_{i}+r a \leq W$

$z_{i} \in\{0,1\}, \quad \forall i \in V$,

$a \in\{0,1\}$.

The formulation of the pricing subproblem is related to a vector of dual variables $\pi$ corresponding to constraints (3b) of the setcover reformulation. A binary variable $z_{i}$ indicates whether item $i$ is selected. A binary variable $a$ indicates whether the knapsack contains at least one uncertain-size item or not. Clearly, the feasible solutions to this problem are the feasible subsets $B \in \mathcal{B}$ of items.

\subsection{Dynamic column generation at root node}

The number of variables of the set-cover formulation grows exponentially with the number of items. Hence, it is only possible to generate all of its columns when the number of items is small. Otherwise, with a large number of items, the well-known principle of dynamic column generation can be applied. Dynamic column generation allows the linear relaxation of the set-cover formulation to be solved, as proposed by Dantzig and Wolfe (1960) for linear programs, considering, implicitly, the whole set of columns. An iteration of dynamic column generation starts with solving the linear relaxation of the set-cover formulation restricted to a relatively small subset of columns. The first iteration begins with a column pool constituted of columns corresponding to subsets of $\mathcal{B}$ containing exactly one item, and of columns in the solution provided by the adapted first-fit decreasing heuristic, described below in Section 3.6.1. Solving the restricted master problem with the simplex method provides values of dual variables $\pi_{i}$, for all $i \in V$.

Then, to check whether an improving column exists, reduced cost pricing is performed, by solving PS, for the current values of dual variables. If its optimal objective value is strictly larger than one, the corresponding improving column, with a negative reduced cost, is added to the current restricted master problem, providing a new restricted master problem, and a new iteration of dynamic column generation starts. If not, no improving column exists, and the current feasible solution to the linear relaxation of the setcover formulation is optimal.

$P S$ is a robust variant of the $0-1$ knapsack problem. It can be solved in pseudo-polynomial time, by solving two instances of the 0-1 knapsack problem, as shown below.

Property 1. PS can be solved in $\mathcal{O}(n \cdot W)$, by solving two instances of the 0-1 knapsack problem. 
Proof. First, the value of binary variable $a$ is set to 0 (the bin contains no uncertain-sized item), and the resulting 0-1 knapsack problem is solved in $\mathcal{O}(n \cdot W)$, with the classical dynamic programming algorithm (see Toth, 1980). Second, the same is done for $a=1$ (the bin is allowed to contain at least one uncertain-sized item). Then, one of the two solutions which provides the best value is selected, and it is an optimal solution to PS. Hence, solving PS requires $\mathcal{O}(2 \cdot n \cdot W)=\mathcal{O}(n \cdot W)$ operations.

Note that, by ordering the items such that certain-sized items are followed by uncertain-sized items, and by adapting the classical dynamic programming algorithm (see Toth, 1980) so that, when uncertain-sized items are reached, the knapsack capacity is decreased by $r$, it is possible to solve PS with a single call to this adapted algorithm. However, we use two calls to the Сомво algorithm (Martello, Pisinger, \& Toth, 1999) to deal with PS and solving two knapsack problems.

As its name suggests, the Сомво algorithm is a combination of several techniques. One of the central ideas upon which it relies is that a small subset of items is generally enough to form an optimal solution. Therefore, the Сомво algorithm restricts the search to a subset of items denoted as the core, which is expanded when needed. Besides this, the algorithm makes use of upper and lower bounds, which allow to fathom dominated states in the list-based dynamic programming procedure, and to terminate the search earlier.

When the Сомво algorithm is called to solve the second instance of the 0-1 knapsack problem, it takes advantage of the lower bound provided by the solution to the first one. It generally enables us to solve the robust $0-1$ knapsack subproblem faster than the adapted dynamic programming algorithm. Note that, for a pricing subproblem occurring when $\widetilde{V}=\emptyset$, Сомво is called only one time (for $a=0$ ).

Finally, we use the bound proposed by Farley (1990) to terminate column generation early when

$\left\lceil v_{\mathrm{RMP}}\right\rceil=\left\lceil\frac{v_{\mathrm{RMP}}}{v_{\mathrm{PS}}}\right\rceil$,

where $v_{\mathrm{RMP}}$ is the linear relaxation value of the current restricted master problem and $v_{\mathrm{PS}}$ is the optimal solution value to the current pricing subproblem. This bound is also used by Vance et al. (1994) and Song et al. (2018). It was shown to be effective in reducing tailing off effects.

\subsection{Branching scheme}

Dynamic column generation provides solutions to the linear relaxation of the set-cover formulation. However, its solution generally does not satisfy integrality constraints. When some of the variables of the solution to the linear relaxation of Problem (3) have non-integral values, branching is performed. We use the branching rule described by Ryan and Foster (1981), which relies on the following property.

Property 2. Let $\mathcal{B}_{i, j} \subset \mathcal{B}$ be the set of patterns that contains both items $i$ and $j$. A solution to the linear relaxation of the set-cover formulation contains variables with non-integral values if and only if there exists an item pair $\{i, j\}$ such that: $0<\sum_{\mathcal{B}_{i, j}} \lambda_{B}<1$ (Ryan $\mathcal{E}^{\prime}$ Foster, 1981).

When the solution to the linear relaxation of the set-cover formulation contains variables with non-integral values, we select one of the item pairs $\{i, j\}$ with the most unfeasible value, that is, which minimizes $\left|0.5-\sum_{\mathcal{B}_{i, j}} \lambda_{B}\right|$. At least one such pair is guaranteed to exist, according to Property 2.

Then, two child nodes are created. In the left node, items $i$ and $j$ have to be in the same bin: the branching constraint is
Table 1

Comparison of two node-selection strategies.

\begin{tabular}{llll}
\hline Strategy & \#OPT & Avg. CPU, (s.) & Avg. \#NODES \\
\hline Most unfeasible & 32 & 1337 & 8420 \\
Largest sum & 12 & 2796 & 11444 \\
\hline
\end{tabular}

$z_{i}=z_{j}$. In the right node, items $i$ and $j$ have to be in different bins: the branching constraint is $z_{i}+z_{j} \leq 1$. In each of the two nodes, the variables of the restricted master problem corresponding to columns which do not satisfy its branching constraints are removed. Each branching constraint is directly added to the subproblem of its node, so that such columns are not generated again. The problem of each node is solved by column generation, and branching occurs as with the root node. The search tree is explored by a depth-first search procedure with restart, in which the bestbound node is selected periodically for a new start of depth-first search.

In Vance et al. (1994), a different item pair selection strategy is proposed: one of the $\{i, j\}$ with the largest total size, but lower than the bin capacity, is selected for branching. We performed numerical experiments with the 50 difficult AI instances introduced by Delorme et al. (2016) to compare this node selection strategy with the one we propose. These instances do not allow free space in any optimal solution. The time limit was set to $3600 \mathrm{~s}$. Results are reported in Table 1 . Column "Strategy" provides the node-selection strategy. Column "Avg. CPU, (s.)" gives the average solution time in seconds. Column "\#OPT" provides the number of proven optimal solutions obtained. Column "\#NODES" gives the average number of opened nodes in the search tree.

The "Most unfeasible" strategy of the proposed branch-and-price algorithm shows faster convergence than the "Largest sum" strategy with these instances. These instances require the exploration of a relatively large search tree, and the choice of this strategy is therefore important with them. In the case of a non-optimal solution, only one extra bin was always used.

\subsection{Subproblems after branching}

Imposing branching constraints $z_{i}=z_{j}$ on pairs $\{i, j\}$ of items in a $0-1$ robust knapsack subproblem preserves its structure. Each of these constraints ensure that either both or none of the items of its pair are selected. Hence, items $i$ and $j$ can be merged into an item of weight $w_{i}+w_{j}$ and value $\pi_{i}+\pi_{j}$. The resulting item has an uncertain size if $i$ or $j$ has an uncertain size, otherwise it is a certain-size item. This subproblem is denoted as $P S^{(l e f t)}$ and it is equivalent to $P S$.

However, imposing branching constraints $z_{i}+z_{j} \leq 1$ on pairs $\{i, j\}$ of items (conflict constraints, also called disjunctive constraints) to a 0-1 robust knapsack subproblem alters its structure. It turns the subproblem into a robust 0-1 knapsack problem with conflict constraints on items, denoted as $P S^{(\text {right })}$. The non-robust variant of this problem is referred in the literature to as $0-1$ knapsack problem with conflicts. The $0-1$ knapsack problem with conflicts is strongly NP-hard, and the more general robust variant is therefore also strongly NP-hard. Property 1 can be straightforwardly adapted to $P S^{(\text {right })}$, which is addressed by solving two instances of the 0-1 knapsack problem with conflicts. The optimal solution value to the first $P S^{(\text {right })}$ instance (for $a=0$ ), is used as a lower bound for the second instance (for $a=1$ ). Note that, for a pricing subproblem occurring when $\widetilde{V}=\emptyset$, this algorithm is called only one time (for $a=0$ ).

We solve the 0-1 knapsack problem with conflicts with the dedicated branch-and-bound algorithm by Sadykov and Vanderbeck (2013), which combines the classic depth-first search based branch-and-bound algorithm for the 0-1 knapsack problem by 
Table 2

Impact of the dominance rule.

\begin{tabular}{llll}
\hline Dominance rule & \#OPT & Avg. CPU, (s.) & Avg. \#NODES \\
\hline Yes & 32 & 1337 & 8420 \\
No & 24 & 1879 & 6427 \\
\hline
\end{tabular}

Kellerer et al. (2004) with the enumeration algorithm for solving the maximum clique problem (or the independent set problem) by Östergård (2002). Upper bounds are computed at each node of the search tree by solving the linear relaxation of the residual knapsack problem, ignoring conflict constraints, with the well-known greedy algorithm for the $0-1$ knapsack problem. This algorithm sorts the items in decreasing order of utility (i.e. the ratio value over weight), and then proceeds to insert them into the knapsack. A limit is set on the number of nodes, which is active only if the current primal bound is greater than one, that is, if an improving column has been found. The branch-and-bound terminates if this limit is reached and if one improving column is available. Otherwise, the solving process goes on, to obtain an improving column, or to prove that no such column exists.

Finally, we introduce the following dominance rule between items for $P S^{(\text {right })}$, which is an extension of classical dominance rules.

Property 3. Let $C_{i} \subset V$ be the set of items in conflict with $i \in V$, i.e., for each $j \in C_{i}$ we have $x_{i}+x_{j} \leq 1$. If the following four conditions hold for any $j \in C_{i}$, then item $j$ is dominated by item $i$, and can be removed from the instance of the robust 0-1 knapsack problem with conflicts:

1. $i \in \widetilde{V} \wedge j \in \widetilde{V}$ or $i \notin \widetilde{V} \wedge j \notin \widetilde{V}$ or $i \notin \widetilde{V} \wedge j \in \widetilde{V}$,

2. $\pi_{i} \geq \pi_{j}$,

3. $w_{i} \leq w_{j}$,

4. $C_{i} \backslash\{j\} \subseteq C_{j} \backslash\{i\}$.

Proof. We show that, if the conditions are verified, any feasible solution $s$ containing item $j$ can be substituted by another solution $s^{\prime}$ in which item $j$ is replaced by item $i$, such that the value of $s^{\prime}$ is greater than or equal to the value of $s$.

First, solution $s^{\prime}$ is feasible, regarding (1) the slack space $r$ required in the presence of at least one uncertain item, as $i \in \widetilde{V} \wedge j \in$ $\widetilde{V}$ or $i \notin \widetilde{V} \wedge j \notin \widetilde{V}$ or $i \notin \widetilde{V} \wedge j \in \widetilde{V}$, (2) the total item size, as $w_{i} \leq w_{j}$, and (3) the conflicts between items, as $C_{i} \backslash\{j\} \subseteq C_{j} \backslash\{i\}$. Second, solution $s^{\prime}$ has a value greater than or equal to solution $s$ since $\pi_{i} \geq \pi_{j}$.

Hence, if the conditions are verified, it is not necessary to consider any solution containing $j$, which can be removed from the instance of the robust 0-1 knapsack problem with conflicts.

This dominance rule allows to eliminate some items of an instance of $P S^{(\text {right })}$ in a preprocessing phase. It is not mentioned in the preceding studies by Vance et al. (1994) and Sadykov and Vanderbeck (2013), which uses the same branching rule, that conducts to a non-robust 0-1 knapsack problem with conflicts as subproblem.

We performed numerical experiments with the 50 difficult AI instances introduced by Delorme et al. (2016) to evaluate the impact of the proposed dominance rule. They do not allow free space in any optimal solution. The time limit was set to 3600 seconds. Results are reported in Table 2. Column "Dominance rule" indicates whether the dominance rule is used or not. Column "Avg. CPU, (s.)" gives the average solution time in seconds. Column "\#OPT" provides the number of proven optimal solutions obtained. Column "\#NODES" gives the average number of opened nodes in the search tree.

The proposed dominance rule enables a faster convergence for the algorithm, while exploring a larger number of nodes, as sub- problems are solved faster after the reduction of the number of items they contain, and this reduction is done efficiently. In the case of a non-optimal solution, only one extra bin was always used.

\subsection{Primal heuristics}

We use the following primal heuristics, which have an important role in the efficiency of the proposed branch-and-pricealgorithm, because they generally provide optimal or near optimal solutions earlier than the branching procedure would.

\subsubsection{Adapted first-fit decreasing heuristic}

The FFD (First-Fit Decreasing) heuristic for BP can be stated as follows: items are sorted by non-increasing weights, and placed into the first bin in that order where it is possible. If no bin with enough free space is available, a new bin is used. FFD uses no more that $11 / 9 O P T+1$ bins for $B P$, where $O P T$ denotes the optimal number of required bins, as shown in Yue (1990).

FFD is simply adapted to $R B P_{1}(r)$ as follows: first, $F F D$ is applied to uncertain-sized items only, with the capacity of the used bins decreased by $r$, producing a partial solution; second, FFD is applied to certain-sized items, using the residual bin capacities of the partial solution, and using new bins when needed.

The main idea here is to pack together uncertain-sized items, as the presence of only one uncertain-sized item in a bin decreases its capacity by $r$. Hence, in an optimal solution, uncertain-sized items tend to be packed together as much as possible. The adapted FFD for $R B P_{1}(r)$ is used to initiate the branch-and-price algorithm with a primal solution, providing both a primal bound and columns for the first restricted master problem.

\subsubsection{Integer programming based heuristics}

We use all the integer programming based heuristics provided with the SCIP Optimization Suite, upon which our branch-andprice algorithm is implemented. Only three of the most efficient ones with the proposed formulation are presented here, for the sake of brevity. They are executed repetitively as new columns are generated during the branch-and-price algorithm, with the aim of constructing integer solutions using the pool of columns available at the current node.

Simple rounding Given a feasible solution to the linear relaxation of the set-cover formulation, simple rounding rounds up each variable to obtain a feasible integer solution.

One-opt One-opt starts from a feasible solution to the set-cover formulation, for example provided by Simple Rounding, and tries to improve it, by shifting some values of the variables from 1 to 0 whenever possible.

ZI Round Given a feasible solution $\lambda$ to the linear relaxation of the set-cover formulation, ZI Round defines the fractionality for each variable $\lambda_{B}$ as $Z I\left(\lambda_{B}\right)=\min \left\{\lambda_{B}-\left\lfloor\lambda_{B}\right\rfloor,\left\lceil\lambda_{B}\right\rceil-\lambda_{B}\right\}$ and the fractionality of the solution as $\operatorname{ZI}(\lambda)=\sum_{B \in \mathcal{B}} Z I\left(\lambda_{B}\right)$. The goal of ZI Round is to identify the variables that can be rounded to improve $Z I(\lambda)$ until the integer infeasibility becomes 0 at which point an integral solution has been found. Therefore, each variable $\lambda_{B}$ is rounded in the direction that reduces $\operatorname{ZI}\left(\lambda_{B}\right)$ the most. In the case of a tie, the variable is rounded down. The ZI Round heuristic is described in detail by Wallace (2010).

\section{Numerical experiments}

We conduct, with three main purposes, numerical experiments based on standard instances of $B P$, from which we construct instances with uncertain-size items. First, we explore numerically the tractability of these problems by the proposed branch-andprice algorithm. Second, we estimate numerically the costs of robustness, in terms of the average additional numbers of required 
Table 3

Results of the proposed branch-and-price algorithm with $\operatorname{RBP}_{1}(r), 870$ runs.

\begin{tabular}{|c|c|c|c|c|c|c|c|c|}
\hline $\begin{array}{l}\tilde{V},(\%) \\
\text { with } \mathrm{GAP}=1\end{array}$ & $r$ & Min. CPU, (s.) & Avg. CPU, (s.) & Max. CPU, (s.) & Std. CPU, (s.) & Avg. \#BIN & \#OPT, (\%) & \#SOL, (\%) \\
\hline \multirow[t]{4}{*}{$10 \%$} & All & 0.09 & 52.28 & 616.78 & 161.45 & 39.69 & $92.3 \%$ & $7.7 \%$ \\
\hline & $0.2 \mathrm{~W}(290)$ & 0.11 & 38.46 & 605.82 & 137.26 & 38.52 & $94.83 \%$ & $5.17 \%$ \\
\hline & $0.3 W(290)$ & 0.1 & 40.26 & 616.78 & 141.86 & 39.44 & $94.14 \%$ & $5.86 \%$ \\
\hline & $0.4 \mathrm{~W}(290)$ & 0.09 & 78.1 & 603.47 & 195.99 & 41.1 & $87.93 \%$ & $12.07 \%$ \\
\hline \multirow[t]{4}{*}{$30 \%$} & All & 0.03 & 55.19 & 601.78 & 168.88 & 44.2 & $91.26 \%$ & $8.74 \%$ \\
\hline & $0.2 W(290)$ & 0.06 & 63.11 & 601.78 & 179.58 & 40.64 & $90 \%$ & $10 \%$ \\
\hline & $0.3 W(290)$ & 0.08 & 38.08 & 600.21 & 140.7 & 43.71 & $94.14 \%$ & $5.86 \%$ \\
\hline & $0.4 \mathrm{~W}(290)$ & 0.03 & 64.37 & 600.21 & 182.4 & 48.26 & $89.66 \%$ & $10.34 \%$ \\
\hline \multirow[t]{4}{*}{$50 \%$} & All & 0.02 & 64.59 & 600.88 & 182.2 & 48.63 & $89.66 \%$ & $10.34 \%$ \\
\hline & $0.2 W(290)$ & 0.05 & 65.43 & 600.02 & 182.21 & 42.68 & $89.66 \%$ & $10.34 \%$ \\
\hline & $0.3 W(290)$ & 0.02 & 62.53 & 600.88 & 179.7 & 47.76 & $90 \%$ & $10 \%$ \\
\hline & $0.4 \mathrm{~W}(290)$ & 0.02 & 65.8 & 600.01 & 185.27 & 55.44 & $89.31 \%$ & $10.69 \%$ \\
\hline All & All & 0.02 & 57.35 & 616.78 & 171.07 & 44.17 & $91.07 \%$ & $8.93 \%$ \\
\hline
\end{tabular}

bins, for the three proposed robust approaches. Third, we perform simulations of solution feasibility under various scenarios generated with different probability distributions for increasing variations of uncertain sizes. Last, an evaluation of the ability of the proposed branch-and-price algorithm to deal with $B P$, using as a reference results found in the literature, is provided in Appendix B.

The branch-and-price algorithm is implemented in $C$ on top of the SCIP Optimization Suite 7.0.2. IBM CPLEX 12.8 is used as the linear programming solver. All numerical experiments were performed on an Intel Core i7-6700HQ CPU at $2.6 \mathrm{GHz}$ with $4 \mathrm{~GB}$ of RAM. The time limit was set to ten minutes of running time per instance. For the branch-and-bound algorithm which solves the knapsack problem with conflicts, the limit on the number of nodes, active only when an improving column has been found, is set to $10000|V|$. Depth first search is restarted from the best-bound node every 100 nodes. Average results are reported here, but full results are available at: https://tinyurl.com/y6myu7uo.

\subsection{Adaptation of the $B P$ instances to $R B P_{1}(r), R B P_{\infty}(r)$ and $R B P_{r r}(\alpha)$}

We considered all the most used $B P$ benchmark sets from the literature, and selected those where item sizes are sufficiently lower than bin capacity, to be turned into feasible robust instances. $B P$ instances from the following studies were adapted to $R B P_{1}(r)$, $R B P_{\infty}(r)$ and $R B P_{r r}(\alpha)$.

Falkenauer (1996) We use the 80 instances from the set Falkenauer $T$, which have between 60 and 501 items, a bin capacity equal to 1000 , and item sizes in the range $\{250, \cdots, 499\}$.

Scholl, Klein, and Jürgens (1997) We use the 10 instances from the set Scholl 3. Their number of items is uniformly distributed between 50 and 500 . The bin capacity is equal to 100,000 . Item sizes are in the range $\{20,000, \cdots, 35,000\}$.

Schwerin and Wäscher (1997) We use two sets of 100 instances, one with 100 items, Schwerin 1, the other with 120 items, Schwerin 2 , and a bin capacity equal to 10,000 . Item sizes are in the range $\{150, \cdots, 200\}$.

Let $P$ stand for the probability for an item to have an uncertain size. We set $P=0.1$ to generate 290 instances, in which a binary flag is simply added to indicate whether an item size is uncertain or not, then $P=0.3$ to generate 290 others and finally $P=0.5$ to generate the last 290. In the following, these instances are used for non-robust $B P$, treating for this problem uncertain sizes as certain, as well as for the robust approaches $R B P_{1}(r), R B P_{\infty}(r)$ and $R B P_{r r}(\alpha)$, for which $r$ or $\alpha$ is considered as a separate parameter.

\subsection{Results of the proposed branch-and-price algorithm for the robust variants of $B P$}

InTables 3-5, we report aggregated results obtained by the proposed branch-and-price algorithm for $R B P_{1}(r), R B P_{\infty}(r)$ and
$R B P_{r r}(\alpha)$, respectively. Results are grouped by percentage of uncertain-size items and by value of $r$ or $\alpha$. Each row provides aggregated results with 290 instances.

Column " $\widetilde{V},(\%)$ " indicates the percentage of uncertain-size items. Column " $r$ " or " $\alpha$ " provides the minimum value of the robustness indicator. The four other columns give aggregated values for the corresponding group of instances. Column "Min. CPU, (s.)" gives the minimum solution time in seconds. Column "Avg. CPU, (s.)" gives the average solution time in seconds. Column "Max. CPU, (s.)" gives the maximum solution time in seconds. Column "Std. CPU, (s.)" gives the standard deviation of solution time in seconds. Column "Avg. \#BIN" indicates the average number of bins in the best found solutions. Column "\#OPT, (\%)" provides the percentage of proven optimal solutions obtained. Column "\#SOL, (\%) with $\mathrm{GAP}=1$ " gives the percentage of solutions with an absolute gap of one with the best known lower bound at the end of the optimization process.

In every case, the proposed algorithm either converged to a proven optimal solution or obtained a solution with an absolute gap of one to the best known lower bound. Hence, for each row, the sum of the two last columns equals 100 percent. As the proposed algorithm always obtains an optimal solution with $R B P_{\infty}(r)$ and $R B B_{r r}(\alpha)$, the last column is omitted in Tables 4 and 5 .

\subsubsection{Results of the proposed branch-and-price algorithm with $R B P_{1}(r)$}

In Table 3, we report average results of the proposed branchand-price algorithm with $R_{B P}(r)$, aggregated by percentage of uncertain-size items and by value of $r$.

With $R B P_{1}(r)$, the proposed branch-and-price algorithm obtains an optimal solution $91 \%$ of the times. Its average running time is less than one minute, and its running time is greater than one minute for $37 \%$ of the runs.

At the end of the optimization process, the absolute gap between the rounded-up lower bound and the best-found integer feasible solution value, within the time limit of ten minutes, is always lower than or equal to one. This means that either one extra bin is used, compared to an optimal solution, or that the solution is optimal, but the rounded-up lower bound is one unit below. In the latter case, proving optimality requires branching to increase the lower bound, which may be computationally expensive, and generally can't be achieved within the time limit of 600 seconds. Only nine such instances were solved within this time limit.

\subsubsection{Results of the proposed branch-and-price algorithm with $R B P_{\infty}(r)$}

In Table 4 , we report average results of the proposed branchand-price algorithm with $R B P_{\infty}(r)$, aggregated by percentage of uncertain-size items and by value of $r$. 
Table 4

Results of the proposed branch-and-price algorithm with $R B P_{\infty}(r), 870$ runs.

\begin{tabular}{|c|c|c|c|c|c|c|c|}
\hline$\widetilde{V},(\%)$ & $r$ & Min. CPU, (s.) & Avg. CPU, (s.) & Max. CPU, (s.) & Std. CPU, (s.) & Avg. \#BIN & \#OPT, (\%) \\
\hline \multirow[t]{4}{*}{$10 \%$} & All & 0.04 & 2.47 & 56.2 & 4.68 & 42.04 & $100 \%$ \\
\hline & $0.2 \mathrm{~W}(290)$ & 0.11 & 2.65 & 21.74 & 4.7 & 40.37 & $100 \%$ \\
\hline & $0.3 W(290)$ & 0.04 & 2.37 & 55.7 & 4.15 & 41.81 & $100 \%$ \\
\hline & $0.4 \mathrm{~W}(290)$ & 0.06 & 2.4 & 56.2 & 5.15 & 43.94 & $100 \%$ \\
\hline \multirow[t]{4}{*}{$30 \%$} & All & 0 & 1.54 & 180.43 & 8.47 & 52.25 & $100 \%$ \\
\hline & $0.2 \mathrm{~W}(290)$ & 0.05 & 1.65 & 20.73 & 3.06 & 46.62 & $100 \%$ \\
\hline & $0.3 W(290)$ & 0.02 & 2.47 & 180.43 & 14.26 & 51.83 & $100 \%$ \\
\hline & $0.4 \mathrm{~W}(290)$ & 0 & 0.48 & 4.99 & 1.06 & 58.31 & $100 \%$ \\
\hline \multirow[t]{4}{*}{$50 \%$} & All & 0 & 0.37 & 16.59 & 0.93 & 65.3 & $100 \%$ \\
\hline & $0.2 \mathrm{~W}(290)$ & 0.01 & 0.77 & 16.59 & 1.51 & 53.1 & $100 \%$ \\
\hline & $0.3 W(290)$ & 0.01 & 0.31 & 1.08 & 0.19 & 63.04 & $100 \%$ \\
\hline & $0.4 \mathrm{~W}(290)$ & 0 & 0.04 & 1.43 & 0.11 & 79.77 & $100 \%$ \\
\hline All & All & 0 & 1.46 & 180.43 & 5.68 & 53.2 & $100 \%$ \\
\hline
\end{tabular}

Table 5

Results of the proposed branch-and-price algorithm with $R B P_{r r}(\alpha), 870$ runs.

\begin{tabular}{|c|c|c|c|c|c|c|c|}
\hline$\widetilde{V},(\%)$ & $\alpha$ & Min. CPU, (s.) & Avg. CPU, (s.) & Max. CPU, (s.) & Std. CPU, (s.) & Avg. \#BIN & \#OPT, (\%) \\
\hline \multirow[t]{4}{*}{$10 \%$} & All & 0.09 & 3.01 & 25.23 & 4.91 & 38.2 & $100 \%$ \\
\hline & $0.2 \mathrm{~W}(290)$ & 0.13 & 2.71 & 22.84 & 4.49 & 37.88 & $100 \%$ \\
\hline & $0.3 W(290)$ & 0.09 & 3.15 & 20.97 & 5.04 & 38.13 & $100 \%$ \\
\hline & $0.4 \mathrm{~W}(290)$ & 0.13 & 3.16 & 25.23 & 5.17 & 38.57 & $100 \%$ \\
\hline \multirow[t]{4}{*}{$30 \%$} & All & 0.03 & 2.78 & 28.34 & 4.63 & 40.45 & $100 \%$ \\
\hline & $0.2 \mathrm{~W}(290)$ & 0.1 & 2.92 & 28.34 & 4.89 & 39.38 & $100 \%$ \\
\hline & $0.3 W(290)$ & 0.03 & 2.87 & 27.33 & 4.85 & 40.44 & $100 \%$ \\
\hline & $0.4 \mathrm{~W}(290)$ & 0.07 & 2.55 & 19.36 & 4.09 & 41.52 & $100 \%$ \\
\hline \multirow[t]{4}{*}{$50 \%$} & All & 0.02 & 2.17 & 21.32 & 3.26 & 42.68 & $100 \%$ \\
\hline & $0.2 \mathrm{~W}(290)$ & 0.06 & 2.53 & 21.32 & 3.96 & 40.83 & $100 \%$ \\
\hline & $0.3 W(290)$ & 0.04 & 2.25 & 15.89 & 3.36 & 42.61 & $100 \%$ \\
\hline & $0.4 \mathrm{~W}(290)$ & 0.02 & 1.72 & 11.67 & 2.17 & 44.61 & $100 \%$ \\
\hline All & All & 0.02 & 2.65 & 28.34 & 4.34 & 40.44 & $100 \%$ \\
\hline
\end{tabular}

With $R B P_{\infty}(r)$, the proposed branch-and-price algorithm always obtains an optimal solution, in less than $2 \mathrm{~s}$ on average. The algorithm requires only six times more than $30 \mathrm{~s}$ to converge, with a maximum solving time at $180 \mathrm{~s}$. The gap between the roundedup value of linear relaxation at root node and the optimal integer solution value is always equal to zero.

\subsubsection{Results of the proposed branch-and-price algorithm with $R B P_{r r}(\alpha)$}

In Table 5, we report average results of the proposed branchand-price algorithm with $R B P_{r r}(\alpha)$, aggregated by percentage of uncertain-size items and by value of $\alpha$.

With $R B P_{r r}(\alpha)$, the proposed branch-and-price algorithm always obtains an optimal solution, in less than $3 \mathrm{~s}$ on average. The algorithm always requires less than $30 \mathrm{~s}$ to converge. The gap between the rounded-up value of linear relaxation at root node and the optimal integer solution value is always equal to zero.

\subsection{Numerical evaluation of the extra cost for robustness}

In the following, the extra cost for robustness is measured as the percentage of extra-bins required in the best found robust solution, for the considered robust approach, compared to the best found non-robust $B P$ solution, to the same base instance. Concerning the $B P$ solutions, 868 out of 870 are optimal, and the two others may be optimal, or use at most one extra bin.

\subsubsection{Extra costs for robustness with $R B P_{1}(r)$ and $R B P_{\infty}(r)$}

In Fig. 1, we report the increase of the number of bins in percentages, compared to the non-robust case, for $R B P_{1}(r)$ and $R B P_{\infty}(r)$, with a percentage of uncertain-size items varying from $10 \%, 30 \%$ to $50 \%$, and $r$ varying by $0.2 W, 0.3 W$ and $0.4 W$.

The extra cost for robustness ranges from $4 \%$ to $146 \%$ of extra bins, depending on the robust approach, the percentage of

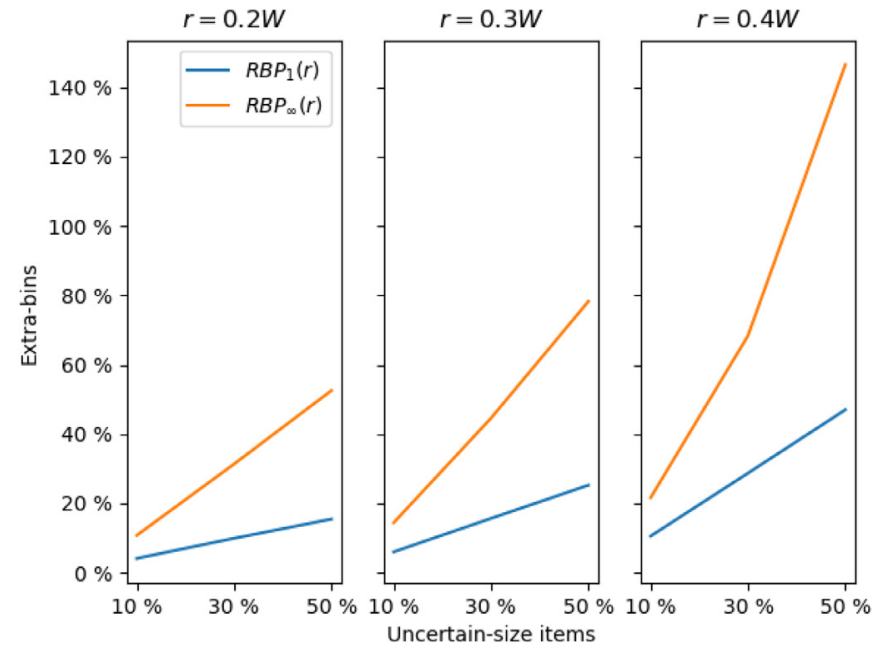

Fig. 1. Increase of the extra cost for robustness, varying the percentage of uncertain-size items and $r$, for $R B P_{1}(r)$ and $R B P_{\infty}(r)$.

uncertain-size items, and $r$. For $R B P_{1}(r)$, this extra cost remains moderate for $\widetilde{V},(\%)=10 \%$, ranging from $4 \%$ to $11 \%$ of extra bins. It grows up to $47 \%$ of extra bins for the maximum values of $r=0.4 \mathrm{~W}$ and $\widetilde{V},(\%)=50 \%$. For $R B P_{\infty}(r)$, this cost sharply increases with $\widetilde{V},(\%)$, particularly for larger value of $r$ : for $r=0.4 W$, it ranges from $22 \%$ to $146 \%$, as $\widetilde{V}$, (\%) increases.

Besides, we observe that the increase of the number of bins, compared to the non-robust case, is on average 2.8 times greater with $R B P_{\infty}(r)$ than with $R B P_{1}(r)$. As can be easily proven, with the same value of the stability radius, and the same uncertain items, the extra cost with the $\ell_{\infty}$ norm is always greater than or equal to the one with the $\ell_{1}$ norm. 

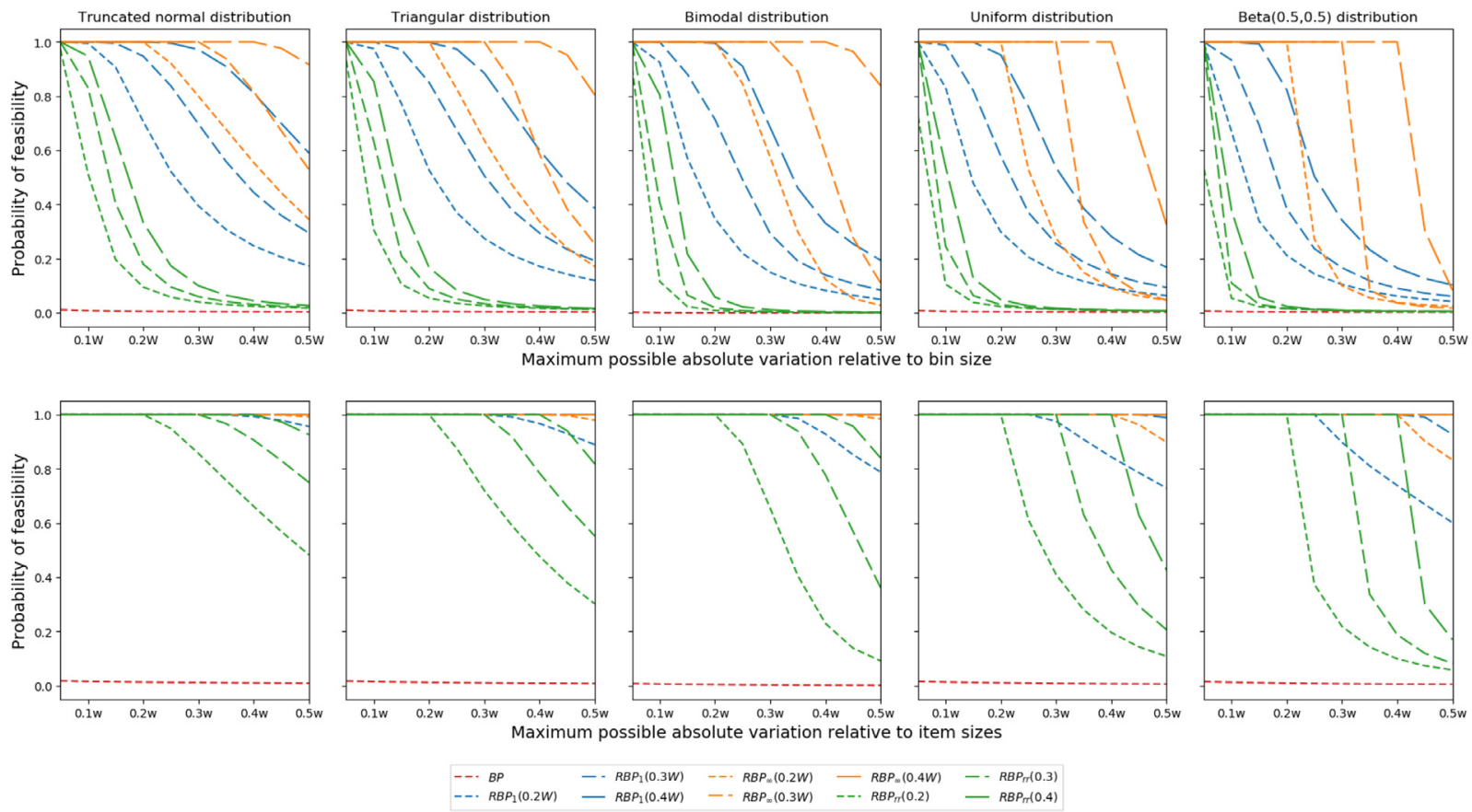

Fig. 2. Simulation results of solution feasibility under five probability distributions, increasing maximum possible absolute variation, relative to bin size or item sizes.

Table 6

Extra cost for robustness increasing relative resiliency, for $R P B_{r r}(\alpha)$.

\begin{tabular}{lll}
\hline$\alpha$ & $\widetilde{V},(\%)$ & Extra bins, (\%) \\
\hline 0.2 & $10 \%$ & $1.97 \%$ \\
& $30 \%$ & $5.80 \%$ \\
0.3 & $50 \%$ & $9.69 \%$ \\
& $10 \%$ & $2.48 \%$ \\
& $30 \%$ & $8.56 \%$ \\
0.4 & $50 \%$ & $14.34 \%$ \\
& $10 \%$ & $3.68 \%$ \\
& $30 \%$ & $11.54 \%$ \\
& $50 \%$ & $19.47 \%$
\end{tabular}

\subsubsection{Extra cost for robustness with $R B P_{r r}(\alpha)$}

In Table 6, we report the increase of the number of bins in percentages, compared to the non-robust case, for $R B P_{r r}(\alpha)$, with a percentage of uncertain-size items equal to $10 \%, 30 \%$ or $50 \%$, when $\alpha$ is set to 0.2 or 0.3 or 0.4 .

Column " $\widetilde{V},(\%)$ " indicates the percent of uncertain-size items. Column " $\alpha$ " indicates the minimum relative resiliency. Column " $E x$ tra bins, (\%)" provides the percent of extra bins required compared to the non-robust case.

With relative resiliency, for $10 \%$ of uncertain items, the extra cost for robustness is almost negligible, under $4 \%$ of extra bins, even for $\alpha=0.4$. It remains under $10 \%$ for $\alpha \leq 0.3$, excepted for $\alpha=0.3$ and $\widetilde{V},(\%)=50 \%$. It reaches a maximum of $19 \%$ for the maximum considered values of $\alpha$ and $\widetilde{V}$, (\%). In the next section, we investigate through simulation the solution feasibility under various scenarios, for the proposed robust approaches, compared to a non-robust one.

\subsection{Simulation of solution feasibility under various scenarios}

We perform numerical simulation of solution feasibility under various scenarios. Random variables describing uncertain-size variations are assumed to be independent and identically distributed, with $[-1,1]$ as probability distribution's support. Five symmetric probability distributions are considered: truncated normal, triangu- lar, bimodal, uniform and beta(0.5, 0.5). A variation is obtained by multiplying the draw of a random variable by a level of variation and by either bin capacity or current item size. Negative variations are converted to 0 . Ten levels of variations are tested, from 0.05 to 0.5 , by increment of 0.05 .

For each of the 870 instances described in Section 4.1, each of the 10 levels of variation, each of the 5 probability distributions, in the case of variations relative to bin capacity and in the case of variations relative to item size, 1000 scenarios are generated, resulting in a total of 87000000 scenarios. The feasibility of each solution obtained by the considered approaches, with the considered values for parameters $r$ or $\alpha$, is evaluated under each of the 100000 scenarios generated for the corresponding instance.

For each probability distribution, in the case of variations relative to bin capacity and in the case of variations relative to item size, we report in Fig. 2 the overall feasibility probability after real item sizes are known for each considered approach, against an increasing level of variation of uncertain sizes.

For a given probability distribution and a given level of variation, this overall feasibility probability of an approach, for a given value of $r$ or $\alpha$, is estimated as the number of scenarios that have a feasible solution, over the total number of scenarios.

We observe that standard BP provides solutions with a feasibility probability always close to 0 , as they don't reserve any free space in the used bins, that could absorb positive variations, and as they use as few bins as possible. Hence, in the case of uncertain sizes, if feasibility is required, a robust approach is a necessity.

Considering variations relative to bin capacity, therefore unrelated to each nominal size, only solutions to $R B P_{\infty}(r)$ have a guarantee of feasibility, as long as any variation is not greater than $r$. Although the cost of robustness with $R B P_{\infty}(r)$ is high (see Section 4.3.1), it is a requirement for this guarantee, according to Property 4. Once $r$ is exceeded, the feasibility probabilities always quickly decrease, as $r$ increases, at a rate that depends on the probability distribution, and which is higher when more extreme values are generated, for example with the $\operatorname{beta}(0.5,0.5)$ distribution. Hence, these solutions are not overly conservative, even in 
the presence of negative variations, as in these simulations, which compensate positive ones.

A solution to $R B P_{1}(r)$ comes with some protection against uncertainty, but generally no guarantee of feasibility. Its feasibility probability is close to one for a range of variation level that depends on the probability distribution and on $r$. With the truncated normal distribution, this probability is greater than 0.7 with a variation level not greater than $r$. For probability distributions generating more extreme values, this probability is lower.

As can be expected, relative resiliency based approaches are inadequate for variations that are not related to nominal item sizes, as they have a low probability of feasibility.

Finally, in the case of variations related to nominal sizes, relative resiliency is a more adequate approach, which offers both a feasibility guarantee and a smaller cost for robustness. In this case, the proposed stability radius based approaches require more bins to offer the same protection against uncertainty.

\section{Conclusion and perspectives}

In this paper, we introduced three robust variants of the $B P$ problem with items of uncertain size. After the assignment of items to bins has been decided, the size of these items can vary. The objective was to minimize the number of bins used, while ensuring the chosen level of robustness. Three indicators of robustness were studied: the stability radius calculated by using the $\ell_{1}$ norm, the stability radius calculated by using the $\ell_{\infty}$ norm, and the relative resiliency, in which possible variations are related to nominal size values. A unique 0-1 linear program was designed, valid for the three robust variants of the $B P$ problem. A set-cover reformulation of the model was done with an exponential number of columns, but a stronger linear relaxation bound. A branch-andprice algorithm with dynamic column generation was developed. Then, a thorough numerical study was performed with thousands of standard benchmark $B P$ instances, in which randomly chosen item sizes were set are uncertain.

We observed that the proposed algorithm is able to obtain an optimal solution to every instance of $R B P_{\infty}(r)$ and $R B P_{r r}(\alpha)$, on average in less than three seconds per instance. It also provided a proven optimal solution to $91 \%$ of $R B P_{1}(r)$ instances, and a solution either optimal or requiring one extra bin to the remaining ones, on average in less than one minute.

We also measured the extra cost for robustness, which ranged from $2 \%$ to $146 \%$ extra bins, depending on the robust approach, the percentage of uncertain items, and the value of $r$ or $\alpha$. Then, we performed simulation of solution feasibility under various scenarios, generated with different probability distributions for increasing variations. We observed that standard $B P$ provides solutions with a feasibility probability always close to 0 . In the case of variations relative to bin capacity, for all the considered robust approaches, feasibility probability always decreases relatively fast, as the level of variation increases. The solutions obtained with $R B P_{\infty}(r)$ have a greater cost for robustness but come with a guarantee of feasibility when any variation is not greater than $r$. With variations observing a truncated normal distribution, a solution to $R B P_{1}(r)$ had a feasibility probability greater than 0.7 with a variation level not greater than $r$. For probability distributions generating more extreme values, this bound was lower. Finally, in the case of variations relative to item sizes, $R B P_{r r}(\alpha)$ offers both a feasibility guarantee and a smaller cost for robustness.

This work offers several perspectives. First, one could consider different robustness models, with for example uncertain bins, in which all item sizes can deviate, or with different kinds of uncertainties, affecting different subsets of items. Second, the proposed branch-and-price algorithm could be improved. To achieve this, a possible research direction is related to the acceleration of the lower bound evaluation, another one to the improvement of this lower bound, and another one to the design of primal heuristics.

\section{Appendix A. Formal definitions}

\section{A1. Definition of stability radius $\rho_{1}$, stability radius $\rho_{\infty}$ and relative resiliency $\rho_{\text {rr }}$}

To evaluate the robustness of a feasible solution, we use the stability radius concept, whose formal definition requires some additional notations to the ones provided in Section 3.1:

- $w=\left(w_{1}, w_{2}, \ldots, w_{n}\right) \in \mathbb{R}_{+}^{n}$, a vector expressing nominal item sizes. For any item $i \in V$ of certain size, nominal and real sizes are assumed to be equal. For any item $i \in \widetilde{V}$ of uncertain size, there may be a difference between nominal and real sizes,

- $\Xi=\left\{\xi \in \mathbb{R}^{n} \mid \xi_{i}=0, i \in V \backslash \widetilde{V}\right\}$, a set of vectors, such that each vector $\xi \in \Xi$ represents a possible scenario of uncertain-size variations,

- $F(w)$, the set of feasible solutions with respect to a given vector $w \in \mathbb{R}_{+}^{n}$,

- $\widetilde{K}=\left\{k \in K \mid V^{k} \cap \widetilde{V} \neq \emptyset\right\}$, the set of bins of a given feasible solution having at least one uncertain item, where $V^{k}$ is the set of items assigned to bin $k$.

The stability radius of a feasible solution $s \in F(w)$ can then be defined as follows (see Sotskov et al., 2006): $\rho(s, w)=\max \{\epsilon \geq 0$ | $\forall \xi \in B(\epsilon), s \in F(w+\xi)\}$, where $B(\epsilon)=\{\xi \in \Xi \mid\|\xi\| \leq \epsilon\}$.

Hence, $\rho(s, w)$ is determined as the value of the radius of the greatest closed ball $B(\cdot)$, called the stability ball which represents uncertain size variations, for which $s$ remains feasible. Any element $\xi$ of $B(\cdot)$ is evaluated based on a given norm $\|\cdot\|$ defining the distance between vectors $w$ and $w+\xi$ (or the amplitude of variations from $w$ ). In this paper, two norms $\ell_{1}\left(\|\cdot\|_{1}\right)$ and $\ell_{\infty}$ $\left(\|\cdot\|_{\infty}\right)$ are considered, where by definition $\|\xi\|_{1}=\sum_{i \in \widetilde{V}}\left|\xi_{i}\right|$ and $\|\xi\|_{\infty}=\max _{\tilde{V}}\left\{\left|\xi_{i}\right|\right\}$. As a consequence, the notations $\rho_{1}$ and $\rho_{\infty}$ will be used for $\ell_{1}$ and $\ell_{\infty}$, respectively.

The integer programming formulation of $R B P_{1}, R B P_{\infty}$ and $R B P_{r r}$ is based on the following three theorems, which are demonstrated by Rossi et al. (2016), by Sotskov et al. (2006) and by Pirogov (2019), respectively.

Theorem 1 (Rossi et al. (2016)). The stability radius $\rho$ for a given feasible solution is calculated as follows: $\rho_{1}=\min _{k \in \widetilde{K}}\left\{W-\sum_{i \in V^{k}} w_{i}\right\}$.

Theorem 2 (Sotskov et al. (2006)). The stability radius $\rho_{\infty}$ for a given feasible solution is calculated as follows: $\rho_{\infty}=$ $\min _{k \in \widetilde{K}}\left\{\frac{W-\sum_{i \in V^{k}} w_{i}}{\left|V^{k} \cap \widetilde{V}\right|}\right\}$.

For some practical applications, a possible limitation of theses approaches is that variations are not related to nominal size values. Therefore, we consider another robust approach, proposed by Pirogov (2019), based on the relative resiliency.

The relative resiliency $\rho_{r r}(s, w)$ refers to the situation where each uncertain-size variation is bounded by an amount that is proportional to the nominal value defined by a coefficient $\alpha>0$. The set of vectors representing possible scenarios of uncertain-size variations is then defined as $\Xi(\alpha, w)=\left\{\xi \in \Xi \mid \xi_{i} \leq \alpha w_{i}, i \in \widetilde{V}\right\}$. In this case, the relative resiliency $\rho_{r r}(s, w)=\max \{\alpha \geq 0 \mid s \in F(w+$ $\xi), \xi \in \Xi(\alpha, w)\}$ is the greatest value of $\alpha$ for which the solution $s$ remains feasible.

Theorem 3 (Pirogov (2019)). The relative resiliency $\rho_{r r}$ for a given feasible solution is calculated as follows: $\rho_{r r}=\min _{k \in \widetilde{K}}\left\{\frac{W-\sum_{i \in V^{k}} w_{i}}{\sum_{i \in V^{k} \cap \widetilde{V}} w_{i}}\right\}$. 


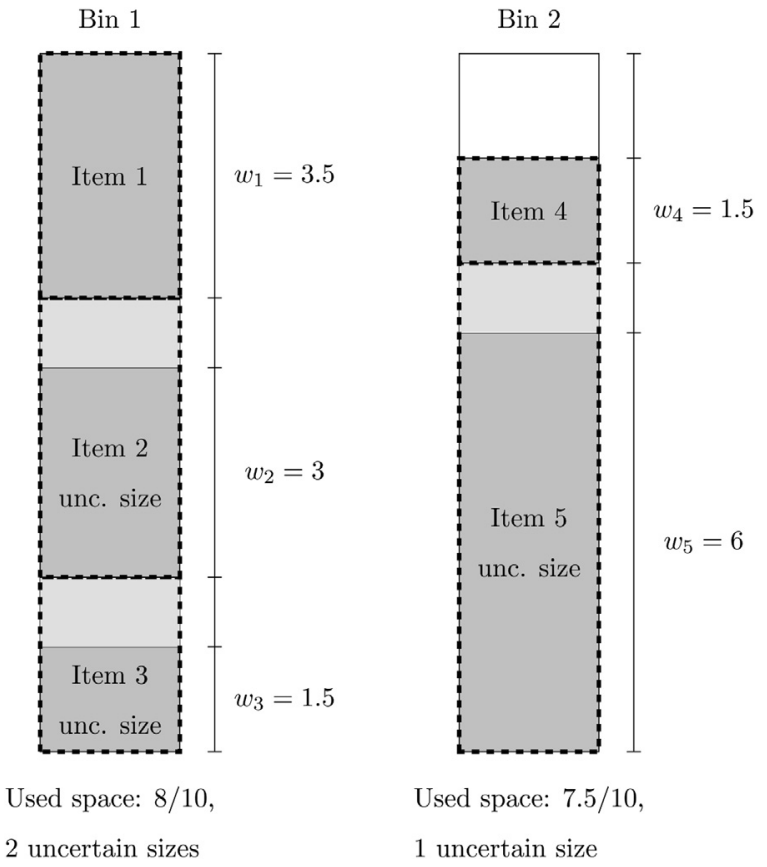

Fig. A1. A robust solution to an instance of $B P$ with $\rho_{\infty}=1$, obtained by reserving one unit of free space for each uncertain size.

\section{A2. Robust bin-packing problems $R B P_{1}(r), R B P_{\infty}(r)$ and $R B P_{r r}(\alpha)$}

We define:

- $R B P_{1}(r)$ (resp. $\left.R B P_{\infty}(r)\right)$ as the problem of obtaining a solution to $B P$ with a stability radius in norm $\ell_{1}$ (resp. $\ell_{\infty}$ ) at least equal to $r \in \mathbb{R}_{+}$using a minimum number of bins.

- $R B P_{r r}(\alpha)$ as the problem of obtaining a solution to $B P$ with a relative resiliency at least equal to $\alpha \in \mathbb{R}_{+}$using a minimum number of bins.

We introduce the following property which states that adding a value $r \in \mathbb{R}_{+}$to each uncertain size ensures that any feasible solution to the resulting instance of $B P$ has a stability radius $\rho_{\infty}$ at least equal to $r$ in the original instance.

Property 4. A given feasible solution of $B P$ has the stability radius $\rho_{\infty}$ at least equal to $r$ iff the following inequality $\sum_{i \in V^{k} \cap \widetilde{V}}\left(w_{i}+r\right)+$ $\sum_{i \in V^{k} \backslash \widetilde{V}} w_{i} \leq W$ holds for any bin $k \in \widetilde{K}$.

Proof. The latter inequality is equivalent to the following one $\sum_{i \in V^{k}} w_{i}+\left|V^{k} \cap \widetilde{V}\right| \cdot r \leq W$, which can be transformed into $r \leq$ $\frac{W-\sum_{i \in V^{k}} w_{i}}{\left|V^{k} \cap \widetilde{V}\right|}$ and, as a consequence, we have $r \leq \min _{k \in \widetilde{K}}\left\{\frac{W-\sum_{i \in V^{k}} w_{i}}{\left|V^{k} \cap \widetilde{V}\right|}\right\}$. Based on Theorem 2, we finally obtain that $r \leq \rho_{\infty}$.

In Fig. $A 1, a$ robust solution to an instance of $B P$ is presented with a total of five items, three uncertain sizes (items 2, 3 and $5)$, a bin capacity of ten, and a stability radius $\rho_{\infty}$ equal to one. Property 4 states that to obtain such a solution, with a stability radius $\rho_{\infty}$ at least equal to one, it is possible to add one to each uncertain size, and to solve the resulting $B P$ instance, as it appears in the figure.

Similarly, the following property states that adding a value $\alpha w_{i}$ to each uncertain size $w_{i}$ for $i \in \widetilde{V}$ ensures that any feasible solution to the resulting instance of $B P$ will have a relative resiliency $\rho_{r r}$ at least equal to $\alpha$ in the original instance.

Property 5. A given feasible solution of BP has a relative resiliency $\rho_{\text {rr }}$ at least equal to $\alpha$ iff the following inequality $\sum_{i \in V^{k} \cap \widetilde{V}}(1+$ $\alpha) w_{i}+\sum_{i \in V^{k} \backslash \widetilde{V}} w_{i} \leq W$ holds for any bin $k \in \widetilde{K}$.
Proof. The latter inequality is equivalent to the following one $\alpha \cdot \sum_{i \in V^{k} \cap \tilde{V}} w_{i}+\sum_{i \in V^{k} \cap \tilde{V}} w_{i} \leq W-\sum_{i \in V^{k} \backslash \widetilde{V}} w_{i}$, which can be transformed into $\alpha \leq \frac{W-\sum_{i \in V^{k}} w_{i}}{\sum_{i \in V^{k} \cap \tilde{V}} w_{i}}$ and, as a consequence, we have $\alpha \leq$ $\min _{k \in \widetilde{K}}\left\{\frac{W-\sum_{i \in V^{k}} w_{i}}{\sum_{i \in V^{k} \cap \widetilde{V}} w_{i}}\right\}$. Based on Theorem 3, we finally obtain that $\alpha \leq$ $\rho_{\text {rr. }}$.

Property 4 (resp. Property 5) implies that a solution to any instance of $R B P_{\infty}(r)$ (resp. $R B P_{r r}(\alpha)$ ) can be obtained by solving an instance of $B P$, in which item sizes are chosen accordingly. However, for $R B P_{1}(r)$, we can't use the same kind of straightforward reduction, as implied by the following Property.

Property 6. A given feasible solution of BP has a stability radius $\rho_{1}$ at least equal to $r$ iff the following inequality $\sum_{i \in V^{k}} w_{i}+r \leq W$ holds for any bin $k \in \widetilde{K}$.

Proof. The latter inequality is equivalent to the following one $r \leq$ $W-\sum_{i \in V^{k}} w_{i}$, which conducts us to $r \leq \min _{k \in \widetilde{K}}\left\{W-\sum_{i \in V^{k}} w_{i}\right\}$. Based on Theorem 1, we finally obtain that $r \leq \rho_{1}$.

Hence, with $R B P_{1}(r)$, any bin containing one uncertain item or more must keep a quantity of unused space at least equal to $r$, which led us to the $0-1$ linear programming formulation of Section 3.1.

\section{Appendix B. Numerical results with BP}

We provide numerical results for $B P$, with instances from the benchmark sets proposed by Falkenauer (1996), Scholl et al. (1997), Wäscher and Gau (1996), Schwerin and Wäscher (1997), Schoenfield (2002) and Delorme et al. (2016). We restrict the report to the subset of 3877 instances (out of 5450) used by Delorme et al. (2016). The maximum amount of time per instance is set to one minute.

In Table $\mathrm{B} 1$, we report comparative numerical results for $B P$ obtained by the proposed branch-and-price algorithm and other branch-and-price algorithms from the literature. In the column "Set", the name of the set of instances is provided. In the column "\#INSTANCE", the number of tested instances for the corresponding set of instances is given. In the columns "\#OPT", "\#OPT Vance", "\#OPT SCIP", “\#OPT Belov", and "\#OPT Song”, the number of instances solved to optimality in less than one minute per instance, by the proposed branch-and-price algorithm, and by the ones by Vance et al. (1994), by Gamrath et al. (2016), by Belov and Scheithauer (2006), and by Song et al. (2018), are provided, respectively. For “\#OPT Vance”, “\#OPT SCIP”, and “\#OPT Belov”, the reported results come from the study by Delorme et al. (2016), in which a benchmark of several algorithms for $B P$ was performed on the same computer, an Intel Xeon CPU at $3.1 \mathrm{GHz}$, with $8 \mathrm{~GB}$ of RAM, faster than the Intel Core i7-6700HQ CPU at $2.6 \mathrm{GHz}$ with 4 GB of RAM that we used. An asterisk in a cell indicates that the algorithm obtained the maximum number of optimal solutions. In Song et al. (2018), results are provided for only six instance sets, and a hyphen "-" in a cell indicates that no results are available for the algorithm.

Globally, the proposed algorithm is able to solve 93\% of the instances of the considered subset in less than one minute per instance. It solves more instances than the previous ones based on the same branching rule: $19 \%$ more instances than the best previous one proposed by Vance et al. (1994). It solves only 6\% less instances than the best known branch-and-price algorithm for $B P$ proposed by Belov and Scheithauer (2006).

Regarding the instances which are not solved within one minute, there are two main difficulties. First, in the case of a large number of items or a large bin capacity, solving the linear relaxation of the master problem at root node may require more than 
Table B1

Comparative results with BP: number of instances solved in at most one minute per instance.

\begin{tabular}{|c|c|c|c|c|c|c|}
\hline Set & \#InStancE & \#OPT & \#OPT Vance & \#OPT SCIP & \#OPT Belov & \#OPT Song \\
\hline Falkenauer U & 74 & 60 & 53 & 18 & $74^{*}$ & - \\
\hline Falkenauer T & 80 & $79^{*}$ & 76 & 35 & 57 & 52 \\
\hline Scholl 1 & 323 & $323^{*}$ & $323^{*}$ & 244 & $323 *$ & - \\
\hline Scholl 2 & 244 & 197 & 204 & 67 & $244^{*}$ & - \\
\hline Scholl 3 & 10 & $10^{*}$ & $10^{*}$ & 0 & $10^{*}$ & 0 \\
\hline Wascher & 17 & 15 & 6 & 0 & $17^{*}$ & 6 \\
\hline Schwerin 1 & 100 & $100 *$ & $100^{*}$ & 0 & $100 *$ & $100^{*}$ \\
\hline Schwerin 2 & 100 & 99 & $100^{*}$ & 0 & $100^{*}$ & $100^{*}$ \\
\hline Hard 28 & 28 & 26 & 11 & 7 & $28^{*}$ & 11 \\
\hline Random 50 & 165 & $165^{*}$ & $165^{*}$ & $165^{*}$ & $165^{*}$ & - \\
\hline Random 100 & 271 & $271^{*}$ & $271^{*}$ & $271^{*}$ & $271^{*}$ & - \\
\hline Random 200 & 359 & $359^{*}$ & 358 & 293 & $359 *$ & - \\
\hline Random 300 & 393 & $393^{*}$ & 387 & 155 & $393 *$ & - \\
\hline Random 400 & 425 & $425^{*}$ & 416 & 114 & $425^{*}$ & - \\
\hline Random 500 & 414 & 412 & 394 & 69 & $414^{*}$ & - \\
\hline Random 750 & 433 & 407 & 99 & 22 & $433^{*}$ & - \\
\hline Random 1000 & 441 & 282 & 62 & 0 & $441^{*}$ & - \\
\hline Total & 3877 & 3623 & 3035 & 1460 & $3854^{*}$ & - \\
\hline
\end{tabular}

one minute. Improving the performance of the proposed branchand-price algorithm would require accelerating the lower bound evaluation. Second, obtaining an integer optimal solution may require too much branching and column generation. A very efficient tailored heuristic such as the one used by Belov and Scheithauer (2006) and introduced by Mukhacheva, Belov, Kartack, and Mukhacheva (2000), may be adapted to $R B P_{1}$ and incorporated into the algorithm, to speed-up the solving process.

\section{References}

Achterberg, T., Berthold, T., \& Hendel, G. (2012). Rounding and propagation heuristics for mixed integer programming. In D. Klatte, H.-J. Lüthi, \& K. Schmedders (Eds.), Operations Research Proceedings 2011 (pp. 71-76). Berlin, Heidelberg: Springer Berlin Heidelberg.

Alem, D. J., Munari, P. A., Arenales, M. N., \& Ferreira, P. A. V. (2010). On the cutting stock problem under stochastic demand. Annals of Operations Research, 179(1), 169-186.

Alfandari, L., Plateau, A., \& Schepler, X. (2015). A branch-and-price-and-cut approach for sustainable crop rotation planning. European Journal of Operational Research, 241(3), 872-879.

Barnhart, C., Johnson, E. L., Nemhauser, G. L., Savelsbergh, M. W. P., \& Vance, P. H. (1998). Branch-and-price: Column generation for solving huge integer programs. Operations Research, 46(3), 316-329.

Battaïa, O., \& Dolgui, A. (2013). A taxonomy of line balancing problems and their solution approaches. International Journal of Production Economics, 142(2), 259-277.

Belov, G., \& Scheithauer, G. (2006). A branch-and-cut-and-price algorithm for one-dimensional stock cutting and two-dimensional two-stage cutting. European Journal of Operational Research, 171(1), 85-106.

Berthold, T. (2008). Heuristics of the branch-cut-and-price-framework SCIP. In J. Kalcsics, \& S. Nickel (Eds.), Operations Research Proceedings 2007 (pp. 31-36). Berlin, Heidelberg: Springer.

Bertsimas, D., \& Sim, M. (2004). The price of robustness. Operations Research, 52(1), $35-53$.

Boysen, N., Fliedner, M., \& Scholl, A. (2007). A classification of assembly line balancing problems. European Journal of Operational Research, 183(2), 674-693.

Cardoen, B., Demeulemeester, E., \& Beliën, J. (2010). Operating room planning and scheduling: A literature review. European Journal of Operational Research, 201(3), 921-932.

Crainic, T. G., Gobbato, L., Perboli, G., \& Rei, W. (2016). Logistics capacity planning: A stochastic bin packing formulation and a progressive hedging meta-heuristic. European Journal of Operational Research, 253(2), 404-417.

Dantzig, G. B., \& Wolfe, P. (1960). Decomposition principle for linear programs. Operations Research, 8(1), 101-111.

Delorme, M., Iori, M., \& Martello, S. (2016). Bin packing and cutting stock problems: Mathematical models and exact algorithms. European Journal of Operational Research, 255(1), 1-20.

Desrosiers, J., \& Lübbecke, M. (2011). Branch-price-and-cut algorithms. In J. Cochran (Ed.), Encyclopedia of Operations Research and Management Science. John Wiley \& Sons.

Falkenauer, E. (1996). A hybrid grouping genetic algorithm for bin packing. Journal of Heuristics, 2(1), 5-30.

Farley, A. A. (1990). A note on bounding a class of linear programming problems, including cutting stock problems. Operations Research, 38(5), 922-923.
Fukasawa, R., Longo, H., Lysgaard, J., De Aragão, M. P., Reis, M., Uchoa, E., \& Werneck, R. F. (2006). Robust branch-and-cut-and-price for the capacitated vehicle routing problem. Mathematical Programming, 106(3), 491-511.

Gamrath, G. (2010). Generic branch-cut-and-price. Technischen Universität Berlin Ph.D. thesis.

Gamrath, G., Fischer, T., Gally, T., Gleixner, A. M., Hendel, G., Koch, T., ... Pfetsch, M. E., et al. (2016). The SCIP Optimization Suite 3.2. Technical Report. Zuse Institute Berlin, Germany.

Horowitz, E., \& Sahni, S. (1974). Computing partitions with applications to the knapsack problem. Journal of the ACM, 21(2), 277-292.

Kellerer, H., Pferschy, U., \& Pisinger, D. (2004). Knapsack problems. Berlin: Springer.

Lamiri, M., Xie, X., Dolgui, A., \& Grimaud, F. (2008). A stochastic model for operating room planning with elective and emergency demand for surgery. European Journal of Operational Research, 185(3), 1026-1037.

Lemaréchal, C. (2007). The omnipresence of lagrange. Annals of Operations Research, 153(1), 9-27.

Martello, S., Pisinger, D., \& Toth, P. (1999). Dynamic programming and strong bounds for the 0-1 knapsack problem. Management Science, 45(3), 414-424.

Mukhacheva, E., Belov, G., Kartack, V., \& Mukhacheva, A. (2000). Linear one-dimensional cutting-packing problems: numerical experiments with the sequential value correction method (SVC) and a modified branch-and-bound method (MBB). Pesquisa Operacional, 20(2), 153-168.

Pirogov, A. (2019). Robust balancing of production lines: MILP models and pre-processing rules. IMT Atlantique, Nantes, France Ph.D. thesis.

Rossi, A., Gurevsky, E., Battaïa, O., \& Dolgui, A. (2016). Maximizing the robustness for simple assembly lines with fixed cycle time and limited number of workstations. Discrete Applied Mathematics, 208, 123-136.

Ryan, D., \& Foster, E. (1981). An integer programming approach to scheduling. In Computer Scheduling of Public Transport Urban Passenger Vehicle and Crew Scheduling (pp. 269-280).

Sadykov, R., \& Vanderbeck, F. (2013). Bin packing with conflicts: A generic branch-and-price algorithm. INFORMS Journal on Computing, 25(2), 244-255.

Scheithauer, G., \& Terno, J. (1997). Theoretical investigations on the modified integer round-up property for the one-dimensional cutting stock problem. Operations Research Letters, 20(2), 93-100.

Schoenfield, J. E. (2002). Fast, exact solution of open bin packing problems without linear programming. Technical Report. US Army Space and Missile Defense Command, Huntsville, Alabama, USA.

Scholl, A., Klein, R., \& Jürgens, C. (1997). Bison: A fast hybrid procedure for exactly solving the one-dimensional bin packing problem. Computers \& Operations Research, 24(7), 627-645.

Schwerin, P., \& Wäscher, G. (1997). The bin-packing problem: A problem generator and some numerical experiments with FFD packing and MTP. International Transactions in Operational Research, 4(5), 377-389.

Song, G., Kowalczyk, D., \& Leus, R. (2018). The robust machine availability problem -Bin packing under uncertainty. IISE Transactions, 50(11), 997-1012.

Sotskov, Y. N., Dolgui, A., \& Portmann, M.-C. (2006). Stability analysis of an optimal balance for an assembly line with fixed cycle time. European Journal of Operational Research, 168(3), 783-797.

Toth, P. (1980). Dynamic programming algorithms for the zero-one knapsack problem. Computing, 25(1), 29-45.

Vance, P. H., Barnhart, C., Johnson, E. L., \& Nemhauser, G. L. (1994). Solving binary cutting stock problems by column generation and branch-and-bound. Computational Optimization and Applications, 3(2), 111-130.

Vanderbeck, F. (2000). On Dantzig-Wolfe decomposition in integer programming and ways to perform branching in a branch-and-price algorithm. Operations Research, 48(1), 111-128. 
Wallace, C. (2010). ZI round, a MIP rounding heuristic. Journal of Heuristics, 16(5), $715-722$.

Wäscher, G., \& Gau, T. (1996). Heuristics for the integer one-dimensional cutting stock problem: A computational study. Operations-Research-Spektrum, 18(3), 131-144.
Yue, M. (1990). A simple proof of the inequality FFD $(L) \leq 11 / 9$ OPT $(L)+1, \forall L$ for the FFD bin-packing algorithm. Acta Mathematicae Applicatae Sinica, 7, 321-331. Östergård, P. R. (2002). A fast algorithm for the maximum clique problem. Discrete Applied Mathematics, 120(1), 197-207. 